\title{
Symmetry-breaking in double gyroid block copolymer film
}

\author{
Seungyun Jo', Haedong Park², Taesuk Jun¹, Kwangjin Kim², Hyunsoo Jung ${ }^{1}$, Sungmin Park ${ }^{3}$, Byeongdu Lee ${ }^{4}$, \\ Seungwoo Lee ${ }^{2,5}$, Du Yeol Ryu ${ }^{1}$
}

${ }^{I}$ Department of Chemical and Biomolecular Engineering, 50 Yonsei-ro, Seodaemun-gu, Yonsei University, Seoul 03722, Republic of Korea, ${ }^{2}$ KU-KIST Graduate School of Converging Science and Technology, Korea University, Seoul 02841, Republic of Korea,

${ }^{3}$ Advanced Materials Division, Korea Research Institute of Chemical Technology, 141 Gajeong-ro, Yuseong-gu, Daejeon 34114, Republic of Korea, ${ }^{4}$ Advanced Photon Source, Argonne National Laboratory, 9700 S. Cass Ave., Lemont, IL 60439, USA,

${ }^{5}$ Department of Integrative Energy Engineering, Department of Biomicrosystem Technology, and KU Photonics Center, Korea University, Seoul 02841, Republic of Korea

\section{blee@anl.gov}

Soft-matter bicontinuous networks find a double gyroid structure from block copolymer (BCP) self-assembly. A gyroid structure composed of dissimilar blocks has proven its potential as a soft crystal, of which the lattice dimension is variable with molecular weight of the polymer. Using an asymmetric polystyrene- $b$-poly(methyl methacrylate) (PS- $b$-PMMA), recently we showed that the self-assembled gyroid films formed via a solvent vapor annealing (SVA) process undergo unique structural distortion due to directional deformation immediately upon deswelling [1]. During the SVA process with PS- $b$-PMMA films, initially transient cylinders developed from the as-cast morphology transform into a cubic gyroid structure in a swollen state. We then observed that upon solvent evaporation the gyroid lattice contracts along the film normal direction while retaining the swollen lateral dimension. The degree of contraction is turned out to be related to the evaporation speed. Rapid and spontaneous deswelling processes lead to triclinic gyroid structures with z-directional contraction ratios $\left(C_{z}\right)$ of 2.5 and 2.0, respectively.

Our X-ray analysis reveals that symmetries of the resulting gyroid structures are partially broken due to the non-affine transformation, eliciting several forbidden reflections such as $\{110\}$ and $\{200\}$ reflections. For further characterization of the symmetry-breaking, we delineate the structural features of noncubic gyroid films by computing electron-density difference maps from grazing incidence small angle X-ray scattering (GISAXS) data. We employed iterative phase retrieval method to solve the phase problem. Level-set approach is accordingly developed to quantitate the structural characteristics of the maps in terms of inversion symmetry-breaking, suggesting its possible application to optical Weyl photonic crystals. This presentation will focus on X-ray data collection and analysis.

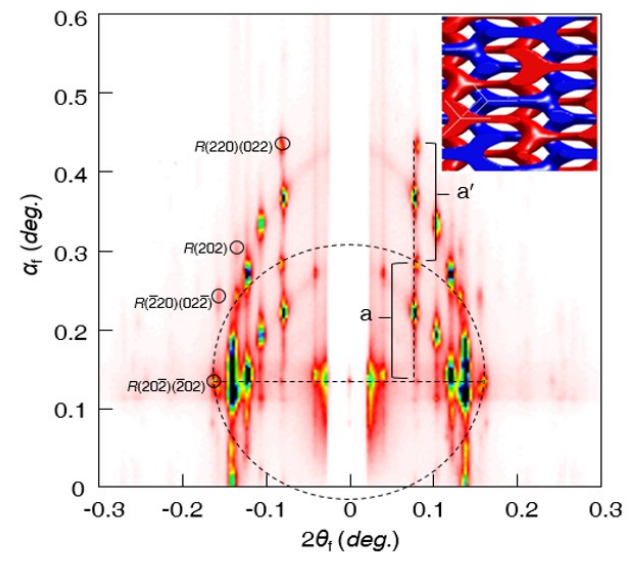

Figure 1. GISAXS data from a $C z=2.5$ sample. Inset is the electron density map calculated from the X-ray data.

[1] Jo, S., Park, H., Jun, T., Kim, K., Jung, H., Park, S., Lee, B., Lee, S., Ryu, D. Y. (2021), Applied Materials Today $23,101006$.

Keywords: block copolymer; GISAXS; electron density map; gyroid; morphology; phase retrieval

GISAXS measurements were performed at Pohang Accelerator Laboratory (Korea) and Advanced Photon Source (APS) at Argonne National Laboratory (US). The APS is supported by the US department of Energy, Office of Basic Energy Sciences, under contract no. DE-AC0206CH11357. This research was supported by Samsung Research Funding \& Incubation Center of Samsung Electronics under Project Number SRFC-MA1801-04.

Acta Cryst. (2021), A77, C137 